\title{
A NOTE ON THE DYNAMICS OF A CLASS OF KOLMOGOROV SYSTEMS
}

\author{
JAUME LLIBRE ${ }^{1}$ AND TAYEB SALHI ${ }^{2}$
}

\begin{abstract}
We characterize the integrability and the non existence of periodic orbits for the 2 dimensional Kolmogorov systems of the form

$$
\begin{aligned}
& \dot{x}=x\left(P_{n}(x, y)+R_{m}(x, y)\right), \\
& \dot{y}=y\left(Q_{n}(x, y)+R_{m}(x, y)\right),
\end{aligned}
$$

where $n$ and $m$ are positive integers and $P_{n}, Q_{n}$ and $R_{m}$ are homogeneous polynomials of degree $n, n$ and $m$, respectively.
\end{abstract}

2010 Mathematics Subject Classification. Primary 34C05, 34A34.

Key words and phrases. Kolmogorov systems, Lotka-Volterra systems, periodic orbits, integrability. 


\section{Introduction AND STATEMENT OF the MAIN RESUlts}

The so called Kolmogorov systems are differential equations of the form

$$
\dot{x}_{i}=x_{i} f_{i}\left(x_{1}, \ldots, x_{n}\right) \quad \text { for } i=1, \ldots, n .
$$

These systems appear in applications that the per unit of change $\dot{x}_{i} / x_{i}$ of the dependent variables $x_{i}=x_{i}(t)$ are given functions $f_{i}\left(x_{1}, \ldots, x_{n}\right)$ of these variables at any time. These systems are also called LotkaVolterra systems because were started to be studied by them in [19] and in [23], respectively. Later on Kolmogorov came, giving some generalisations in [14] and then some authors denote them by Kolmogorov systems.

There are many natural phenomena which can be modeled by the Kolmogorov systems such as mathematical ecology and population dynamics [21], chemical reactions, plasma physics [15], hydrodynamics [3], economics, etc.

Starting with Volterra, mathematicians have been interested in Kolmogorov systems particularly in

- their integrability, i.e. when such differential systems have first integrals (see for instance $[1,2,4,5,6,7,8,17,18,22]$ ), or

- in their periodic orbits (see for example $[9,10,11,13,16,20$, $24,25,26])$.

See also the references quoted in those articles.

In this paper we are interested in studying the integrability and the periodic orbits of the 2-dimensional Kolmogorov systems of the form

$$
\begin{aligned}
& \dot{x}=x\left(P_{n}(x, y)+R_{m}(x, y)\right), \\
& \dot{y}=y\left(Q_{n}(x, y)+R_{m}(x, y)\right),
\end{aligned}
$$

where $n$ and $m$ are positive integers and $P_{n}, Q_{n}$ and $R_{m}$ are homogeneous polynomials of degree $n, n$ and $m$, respectively.

Let $U$ be a non-empty open and dense subset of $\mathbb{R}^{2}$. We say that a non-locally constant $C^{1}$ function $H: U \rightarrow \mathbb{R}$ is a first integral of the polynomial differential system (1) in $U$ if $H$ is constant on the trajectories of the polynomial differential system (1) contained in $U$, i.e. if

$$
\frac{d H}{d t}=\frac{\partial H}{\partial x} x\left(P_{n}(x, y)+R_{m}(x, y)\right)+\frac{\partial H}{\partial y} y\left(Q_{n}(x, y)+R_{m}(x, y)\right)=0,
$$

in the points of $U$. 
We define the trigonometric polynomials

$$
\begin{aligned}
& f(s)=Q_{n}(\cos s, \sin s) \sin ^{2} s+P_{n}(\cos s, \sin s) \cos ^{2} s, \\
& g(s)=\left(Q_{n}(\cos s, \sin s)-P_{n}(\cos s, \sin s)\right) \cos s \sin s .
\end{aligned}
$$

Our main result on the integrability and the periodic orbits of the Kolmogorov system (1) is the following.

Theorem 1. Consider a polynomial Kolmogorov system (1). Then the following statements hold.

(a) If $g(s) \not \equiv 0$ and $m \neq n$, then system (1) has the first integral

$$
\begin{aligned}
H= & \left(x^{2}+y^{2}\right)^{(n-m) / 2} \exp \left((m-n) \int^{\arctan (y / x)} F(s) d s\right) \\
& +(m-n) \int^{\arctan (y / x)} \exp \left((m-n) \int^{u} F(s) d s\right) G(u) d u,
\end{aligned}
$$

where $F(s)=f(s) / g(s)$ and $G(s)=R_{m}(\cos s, \sin s) / g(s)$.

(b) $g(s) \not \equiv 0$ and $m=n$, then system (1) has the first integral

$$
H=\sqrt{x^{2}+y^{2}} \exp \left(-\int^{\arctan (y / x)}(F(s)+G(s)) d s\right) .
$$

(c) If $g(s) \equiv 0$, then system (1) has the first integral $H=y / x$.

(d) System (1) has no periodic orbits.

Theorem 1 is proved in section 2 .

\section{Proof of Theorem 1}

If we write system (1) in polar coordinates $(r, \theta)$ where $x=r \cos \theta$ and $y=r \sin \theta$, then we obtain

$$
\begin{aligned}
& \dot{r}=r^{n+1} f(\theta)+r^{m+1} R_{m}(\cos \theta, \sin \theta), \\
& \dot{\theta}=r^{n} g(\theta),
\end{aligned}
$$

where the functions $f(\theta)$ and $g(\theta)$ are given in (2).

If $g(\theta) \not \equiv 0$ and $m \neq n$ we take as new independent variable the variable $\theta$, then the differential system (5) becomes the differential equation

$$
\frac{d r}{d \theta}=r F(\theta)+r^{m+1-n} G(\theta),
$$

where the functions $F(\theta)$ and $G(\theta)$ are the ones defined in statement (a) of Theorem 1. 
We note that the differential equation (6) is a Bernoulli differential equation, see for more details [12]. Then, doing the change of variables $\rho=r^{n-m}$ the Bernoulli differential equation becomes the linear differential equation

$$
\frac{d \rho}{d \theta}=(n-m)(\rho F(\theta)+G(\theta))
$$

which has the first integral

$$
\begin{aligned}
H= & \rho \exp \left((m-n) \int^{\theta} F(s) d s\right) \\
& +(m-n) \int^{\theta} \exp \left((m-n) \int^{u} F(s) d s\right) G(u) d u,
\end{aligned}
$$

Hence statement (a) of Theorem 1 is proved.

Suppose now that $g(\theta) \not \equiv 0$ and $m=n$. Then the differential equation (6) becomes

$$
\frac{d r}{d \theta}=r(F(\theta)+G(\theta))
$$

which has the first integral

$$
H=r \exp \left(-\int^{\theta}(F(s)+G(s)) d s\right) .
$$

Therefore it follows statement (b) of Theorem 1.

Assume now that $g(\theta) \equiv 0$. Then, from (5) it follows that $\dot{\theta}=0$. So the straight lines trough the origin of coordinates of the differential system (1) are invariant by the flow of this system. Hence, $y / x$ is a first integral of the system, and this completes the proof of statement (c) of Theorem 1.

The equilibrium points of the Kolmogorov system (1) are located at the origin, or on the $x$ or $y$ axes, or in some of the open four quadrants obtained from $\mathbb{R}^{2}$ removing the $x$ and $y$ axes. Since the axes $x$ and $y$ are formed by trajectories of the system (1), surrounding the equilibria located on these axes cannot be periodic orbits. Let $\gamma$ be a periodic orbit surrounding an equilibrium located in one of the open quadrants, and let $h_{\gamma}=H(\gamma)$.

Assume that $g(s) \not \equiv 0$ and $m \neq n$. Then, the curves $H=h$ with $h \in \mathbb{R}$, which are formed by trajectories of the differential system (5), 
can be written as

$$
\begin{aligned}
& r(\theta)=\left[\frac { 1 } { h } \left(\exp \left((m-n) \int^{\theta} F(s) d s\right)\right.\right. \\
& \left.\left.\quad+(m-n) \int^{\theta} \exp \left((m-n) \int^{u} F(s) d s\right) G(u) d u\right)\right]^{\frac{1}{m-n}} .
\end{aligned}
$$

Therefore the periodic orbit $\gamma$ is contained in the curve

$$
\begin{aligned}
& r(\theta)=\left[\frac { 1 } { h _ { \gamma } } \left(\exp \left((m-n) \int^{\theta} F(s) d s\right)\right.\right. \\
& \left.\left.\quad+(m-n) \int^{\theta} \exp \left((m-n) \int^{u} F(s) d s\right) G(u) d u\right)\right]^{\frac{1}{m-n}} .
\end{aligned}
$$

But this curve cannot contain the periodic orbit $\gamma$ contained in one of the open quadrants because this curve at most have a unique point on every ray $\theta=\theta^{*}$ for all $\theta^{*} \in[0,2 \pi)$.

Suppose that $g(s) \not \equiv 0$ and $m=n$. From (8) now the curves $H=h$ with $h \in \mathbb{R}$ can be written as

$$
r(\theta)=h \exp \left(\int^{\theta}(F(s)+G(s)) d s\right) .
$$

So the periodic orbit $\gamma$ must be contained in the curve

$$
r(\theta)=h_{\gamma} \exp \left(\int^{\theta}(F(s)+G(s)) d s\right) .
$$

Again this curve cannot contain the periodic orbit $\gamma$ for the same reason than in the previous case.

Finally assume that $g(s) \equiv 0$. Then since all the straight lines through the origin are formed by trajectories, clearly the system has no periodic orbits. This completes the proof of statement (d) of Theorem 1.

\section{ACKNOWLEDGMENTS}

The first author is partially supported by a MICINN/FEDER grant MTM2008-03437, a CIRIT grant number 2009SGR-410, FP7-PEOPLE -2012-IRSES 316338 and 318999, and ICREA Academia. The second author is partially supported by the Algerian Ministry of Higher Education and Scientific Research. 


\section{REFERENCES}

[1] O.I. BogoyavlenskiJ, Integrable Lotka-Volterra systems, Regul. Chaotic Dyn. 13 (2008), 543-556.

[2] O. Bogoyavlenskij, Y. Itoh and T. Yukawa, Lotka-Volterra systems integrable in quadratures, J. Math. Phys. 49 (2008), 053501, 6 pp.

[3] F.H. Busse, Transition to turbulence via the statistical limit cycle route, Synergetics, Springer-Verlag, Berlin, 1978, p. 39.

[4] L. Cairó And J. LliBre, Phase portraits of cubic polynomial vector fields of Lotka-Volterra type having a rational first integral of degree 2, J. Phys. A 40 (2007), 6329-6348.

[5] L. Cairó, H. Giacomini and J. Llibre, Liouvillian first integrals for the planar Lotka-Volterra system, Rend. Circ. Mat. Palermo (2) 52 (2003), 389418.

[6] T. Carleman, Application de la théorie des équations intégrales linéaires aux systémes d'équations différentielles non linéaires, Acta Math. 59 (1932), 6387.

[7] P. GAO, Hamiltonian structure and first integrals for the Lotka-Volterra systems, Phys. Lett. A 273 (2000), 85-96.

[8] R. Gladwin Pradeep, V.K. Chandrasekar, M. Senthilvelan and M. LAKSHMANAN, On certain new integrable second order nonlinear differential equations and their connection with two dimensional Lotka-Volterra system, J. Math. Phys. 51 (2010), 033519, 23 pp.

[9] L.J. Golaszewski, P. Slawinski and H. Zoladek, Limit cycles in 3D Lotka-Volterra systems appearing after perturbation of Hopf center, Internat. J. Bifur. Chaos Appl. Sci. Engrg. 18 (2008), 3647-3656.

[10] M. Gyllenberg, P. YAN AND Y. WANG, Limit cycles for competitorcompetitor-mutualist Lotka-Volterra systems, Phys. D 221 (2006), 135-145.

[11] M. Gyllenberg, P. YAn and Y. WAng, A 3D competitive Lotka-Volterra system with three limit cycles: a falsification of a conjecture by Hofbauer and So, Appl. Math. Lett. 19 (2006), 1-7.

[12] E. Hairer, S.P. Norsett And G. Wanner, Solving ordinary differential equations I: Nonstiff problems, Berlin, New York: Springer-Verlag, 1993.

[13] X.C. Huang And L. Zhu, Limit cycles in a general Kolmogorov model, Nonlinear Anal. 60 (2005), 1393-1414.

[14] A. Kolmogonov, Sulla teoria di Volterra della lotta per l'esistenza, Giornale dell' Istituto Italiano degli Attuari 7 (1936), 74-80.

[15] G. Laval and R. Pellat, Plasma Physics. Proceedings of Summer School of Theoretical Physics, Gordon and Breach, New York, 1975.

[16] C. Li AND J. LliBRe, The cyclicity of period annulus of a quadratic reversible Lotka-Volterra system, Nonlinearity 22 (2009), 2971-2979.

[17] J. Llibre And C. VAlls, Global analytic first integrals for the real planar Lotka-Volterra system, J. Math. Phys. 48 (2007), 033507, 13 pp.

[18] J. Llibre And C. VAlls, Polynomial, rational and analytic first integrals for a family of 3-dimensional Lotka-Volterra systems, Z. Angew. Math. Phys. 62 (2011), 761-777.

[19] A.J. LotKA, Analytical note on certain rhythmic relations in organic systems, Proc. Natl. Acad. Sci. U.S. 6 (1920), 410-415. 
[20] Z. LU AND Y. LuO, Two limit cycles in three-dimensional Lotka-Volterra systems, Comput. Math. Appl. 44 (2002), 51-66.

[21] R.M. MAY, Stability and Complexity in Model Ecosystems, Princeton New Jersey, 1974.

[22] R. TUdorAn AND A. GÎRBAn, On a Hamiltonian version of a threedimensional Lotka-Volterra system, Nonlinear Anal. Real World Appl. 13 (2012), 2304-2312.

[23] V. Volterra, Lecons sur la Théorie Mathématique de la Lutte pour la vie, Gauthier Villars, Paris, 1931.

[24] Y. WANG, The necessary and sufficient conditions for the existence of periodic orbits in a Lotka-Volterra system, J. Math. Anal. Appl. 284 (2003), 236-249.

[25] Q. WANG, W. HuANG AND B. LI, Limit cycles and singular point quantities for a 3D Lotka-Volterra system, Appl. Math. Comput. 217 (2011), 8856-8859.

[26] D. XIAO AND W. Li, Limit cycles for the competitive three dimensional LotkaVolterra system, J. Differential Equations 164 (2000), 1-15.

1 Departament de Matemàtiques, Universitat Autònoma de Barcelona, 08193 Bellaterra, Barcelona, Catalonia, Spain

E-mail address: jllibre@mat.uab.cat

2 Département de Mathématiques, Université de Bordj Bou Arréridj, Bordj Bou Arréridj 34265, El Anasser, Algeria

E-mail address: salhi3tayeb@yahoo.fr 